\title{
Processes of inference during sentence encoding
}

\author{
MURRAY SINGER \\ University of Manitoba, Winnipeg, Manitoba R3T 2N2 Canada
}

\begin{abstract}
Four experiments were designed to determine whether implicit instruments are "computed" and stored during sentence encoding. Subjects read pairs of related sentences and indicated when they understood the second member of each pair. In Experiment 1, response times were longer for test sentences that, relative to the antecedent, mentioned implicit rather than explicit instruments. Experiment 2 revealed this difference to be stable over a range of reading times and two phases of practice. The results were interpreted as suggesting that the inferences in question are not computed, or at most, are partially drawn during encoding In Experiment 3, the procedure was employed to distinghish four degrees of relation between propositions and the inferences they permit. Experiment 4 examined the agent, patient, and instrument cases. It was shown that subjects need more time to verify both true and false inference tests than to verify their direct counterparts.
\end{abstract}

Recent investigations of comprehension have emphasized the inferences that the language recipient may compute on the basis of a verbal message. By now, evidence has been provided that indicates that sentence or discourse input permits the language recipient to make judgments concerning implied cause and consequence, necessary and possible roles (Clark, 1977), presupposition, logical and pragmatic (i.e., probabilistic) implications of verbs, and numerous other relations. One theme that runs through many of these studies concerns the issue of whether such inferences are computed and stored at the time the input is encoded, or only at a later time, when a reexamination of the stored representation is necessary.

The present study was designed to examine this issue as it applies to verb-entailed roles or cases. To pose the problem in the form of a simple example, it is possible to ask whether the language recipient computes and stores the use of a broom when Sentence 1 (see Table 1) is encountered. (Throughout the article, references to numbered sentences and sentence sets refer to the items contained in Table 1.)

Evidence that this inference is so computed stems from two sources. First, having encountered Sentence 1 in a paragraph, subjects incorrectly report recognizing statements expressing reasonable implications, like Sentence 2 (Johnson, Bransford, \& Solomon, 1973; Thorndyke, 1976). If it is assumed that recognition results only when the test sentence matches a portion

This research was supported by a grant from the National Research Council of Canada. I am grateful to Roy Gabriel, Susan Haviland, and Marcel Just for their helpful suggestions concerning the analysis of these data. I would also like to thank Gail Singer and John Whiteley for their thoughtful remarks about the manuscript. Requests for reprints should be sent to M. Singer, Department of Psychology, University of Manitoba, Winnipeg, Manitoba R3T 2N2, Canada. of the internal representation of the passage, then it may be concluded that the relevant inference had been drawn earlier. Second, cues that express an implicit relation based upon antecedent input have been shown to function as effective prompts for the recall of the antecedent material (Anderson \& Ortony, 1975; Paris

Table 1

Sentences and Sentence Sets

1. The sailor swept the floor in the cabin.

2. The sailor swept the floor with the broom.

3. The millionaire was murdered on a dark and stormy night.

4a. We got some beer out of the trunk.

$4 b$. We checked the picnic supplies.

$4 c$. The beer was warm.

5a. The boy cleared the snow with a shovel.

5 b. The boy cleared the snow from the stairs.

5c. The boy hated working with a shovel.

5d. The shovel was heavy.

6a. The boy cleared the snow with the shovel. (Level 1)

6b. The boy cleared the snow from the stairs. (Level 2)

6c. The boy cleared a path on the stairs. (Level 3)

6d. The boy cleared the bottles from the stairs. (Level 4)

6e. The shovel was heavy. (Test)

7a. The millionaire was murdered on a dark and stormy night.

$7 \mathrm{~b}$. The killer left no clues for the police to trace.

8a. The millionaire died on a dark and stormy night.

8 b. The killer left no clues for the police to trace.

9a. The waiter lit the candle with the match.

$9 \mathrm{~b}$. The waiter lit the candle at the table.

9c. The waiter used a match.

9d. The waiter used a rock.

10a. The dentist filled the tooth painlessly.

10b. The tooth was filled painlessly.

10c. A dentist filled the tooth.

10d. A principal filled the tooth.

11a. The pitcher threw the ball to first base.

11b. The pitcher threw to first base.

11c. The pitcher threw a ball.

11d. The pitcher threw a glove. 
\& Lindauer, 1976; Till, 1977; Paris, Sorkin, \& Pisoni, Note 1). This efficiency might likewise be explained by positing that the inference in question is drawn on input, permitting a match to occur between the prompt and an element of the stored representation. The stored information could then be "read" by the subject. It should be mentioned that while these results may be viewed as consistent with an inference-during-encoding position, it is not suggested that the authors in question unequivocally came to this conclusion.

It is possible, however, to suggest explanations of both the recognition of implicational sentences and the effectiveness of implicit recall prompts that make no reference to inferences at the time of encoding. With regard to the former, it is possible that the subject who incorrectly reports recognizing a test sentence that expresses an implication of an antecedent has computed that implication at the time of the test (Johnson et al., 1973). Singer's (Note 2) subjects, in fact, responded in a postsession questionnaire that they reported recognizing test sentences as long as they were consistent with the gist of antecedent passages. This is consistent with the possibility that recognition of implicit sentences might be based upon inferences at test or retrieval time.

Singer's (Note 2) response latency data also support an inference-at-test position. Subjects needed about $.2 \mathrm{sec}$ longer to both recognize and verify implicit test items, as compared with explicit ones. In a related study, Just and Carpenter (in press) tentatively concluded that the reader who encounters Sentence 3 ("the millionaire was murdered on a dark and stormy night") does not infer the existence of a killer during encoding.

Consider next the effectiveness of implicit recall cues. Suppose that the subject encounters "the sailor swept the floor in the cabin" during acquisition and subsequently receives "broom" as a retrieval prompt. If, at that time, the subject computes the usual function of "broom," the result "to sweep" will provide access to the stored representation, regardless of whether the use of a broom has been inferred during encoding or not. While studies of the phenomenon of encoding specificity and of related effects reveal that such a "backward associative" explanation cannot account for all results related to implicit cued recall (Anderson \& Ortony, 1975; Barclay, Bransford, Franks, McCarrell, \& Nitsch, 1974; Tulving \& Thomson, 1973), these studies do not prove that a mechanism of backward association can never be effective. In this vein, Hayes-Roth and HayesRoth (1977, p. 121) have considered a backward computational model as one possible explanation of the effectiveness, as recall cues, of synonyms of words from acquisition-list sentences. Corbett and Dosher (in press), furthermore, have shown that "hammer" functions as an effective cue even for "the worker pounded the nail with a rock." It is clear that subjects should not infer the use of a hammer when they encounter this antecedent.
In many of the studies that have addressed the question of whether inferences are drawn during encoding, subjects' knowledge concerning prior input has been assessed after a moderate delay. It is somewhat hazardous, however, to draw conclusions about what the reader may have done during encoding, on the basis of such evidence. A fruitful path toward solving this problem is exemplified in the studies of Harris (1974) and Just and Carpenter (1976), who tested their subjects both immediately after encoding and at a later time. It stands to reason that if a particular inference is typically drawn during encoding, there should be strong evidence of it immediately following. With the passage of time, this evidence may be obscured due to the evolution of the resulting internal representation.

The present study, therefore, further examined the problem of the temporal locus of inference processes by testing subjects immediately after sentence encoding. To accomplish this, the procedure of Haviland and Clark (1974) was employed. Those investigators asked their subjects to read one sentence and then to press a key as soon as they understood a closely related second sentence. Their materials consisted of triples of sentences, illustrated by Sentence Set 4 . Subjects read either Sentence $4 \mathrm{a}$ or Sentence $4 \mathrm{~b}$ as the antecedent, followed by test Sentence 4c. Sentence $4 c$ comprised a "direct" test after $4 a$ and an "indirect" test after $4 b$. Haviland and Clark showed that more time is needed to comprehend indirect tests and at tributed this latency difference to the execution of mental processes permitting the discovery, in episodic memory, of an antecedent for the "given" (or presupposed) element of the indirect test sentence.

Haviland and Clark's (1974) procedure constitutes a convenient diagnostic device for inferences during encoding. Consider Sentence Set 5: If the reader infers the use of a shovel when Sentence $5 b$ is encountered, it should take no more time to comprehend $5 \mathrm{~d}$ after $5 b$ (inference) than after $5 a$ (direct). If this inference is not computed or is incompletely computed, inference latencies should exceed direct ones.

The present study examined implications primarily concerning the instrumental case (Fillmore, 1968), that is, concerning the instrument used to perform a specified action. While these implications are pragmatic, the materials were constructed so as to suggest implications with which a large majority of subjects would agree (Singer, Note 3). Four experiments were conducted using these materials. The purpose of the first experiment was to determine whether subjects draw highly probable instrumental inferences in the course of comprehension. The second was designed to follow the time course of inference activities during reading periods of up to $6 \mathrm{sec}$. The third experiment asked whether the present procedure might reflect varying degrees of difficulty in inference processes. Experiment 4 extended the research to cases other than the instrumental and answered some questions about Experiment 1. 


\section{EXPERIMENT 1}

\section{Method}

Subjects. The subjects were 36 male and female undergraduate students of introductory psychology at the University of Manitoba. They participated in the experiment in partial fulfillment of a course requirement. All subjects were native speakers of English.

Materials. The verbal materials consisted of 18 sentence sets, such as Set 5 in Table 1 . Sentences 5a-c functioned as antecedents that were related to the test (Sentence 5d) in different ways. Sentence 5 a constituted a direct antecedent, since "shovel" is explicitly mentioned. In $5 \mathrm{~b}$, on the other hand, the use of a shovel is only implied.

The third antecedent, Sentence 5c, functioned as a control, analogous to one employed by Haviland and Clark (1974, Experiment 2). Ordinarily, indefinite articles such as "a" and "some" introduce new concepts in a discourse, while definite articles, like "the," refer to concepts described earlier. In Sentence 5c, however, the phrase "a shovel" does not specify the existence of a particular shovel. For this reason, additional processing should be needed to reconcile test Sentence $5 \mathrm{~d}$ with antecedent $5 \mathrm{c}$, and control latencies should, therefore, exceed direct ones. If they do not, then possible latency differences between the direct and inference conditions could be attributed to the facilitation of the encoding of that part of the direct test that matches a portion of the antecedent (e.g., the word "shovel").

The inference antecedents were constructed so as to convey instrumental implications with which most subjects would agree. To achieve this aim, reference was made to the norms of Singer (Note 3). Singer asked his subjects to specify the three instruments that they thought were most likely to be used to perform 70 different actions. The actions were expressed in the norming study as verb phrases, like "clear the snow" and "drive the nail." The 18 action phrases examined in Experiments 1-3 had first-choice instruments that were agreed upon by an average of $85.1 \%$ of the subjects $(\mathrm{SE}=1.8 \%)^{1}$

For the purpose of counterbalancing, three test lists were constructed. The lists included one sentence pair (antecedent plus test) from each of the 18 sets, with six sentence pairs in each of the three conditions. Across the three lists, it was possible to pass each set through the three conditions.

Each list included another 18 filler pairs, consisting of analogous direct, inference, and control items, based upon category relations (e.g., antecedent, "The boy watched the trout in the pool"; test, "The fish broke the surface"). The filler materials were not systematically counterbalanced through the lists. Finally, the list was preceded by nine practice items, similar in nature to the experimental and filler pairs. The subjects were not told that these items were included simply for practice.

Procedure. The subjects were randomly assigned to recive one of the three lists. Each list was viewed by 12 subjects. The subjects were tested individually, employing a procedure similar to that described by Haviland and Clark (1974). On each trial, the subject viewed a fixation point in a tachistoscope immediately after pressing the READY button. After $1 \mathrm{sec}$, the antecedent sentence appeared in the top field of the tachistoscope, beginning exactly where the fixation point had occurred. This sentence was viewed at a distance of $52 \mathrm{~cm}$. After $2.5 \mathrm{sec}$, the sentence disappeared and was replaced immediately by the test item in the lower field. The subject was asked to press a RESPONSE button as soon as (s)he felt that the test item was understood. Response latency from the onset of the test sentence was recorded to the nearest $.001 \mathrm{sec}$.

The main point of departure between this plan and that described by Haviland and Clark (1974) was the experimenterpaced $2.5-\mathrm{sec}$ reading period for the antecedent. Haviland and
Clark used a subject-paced interval. It was felt here that it would be desirable to control the reading period, while insuring that the subject had sufficient time to read the antecedent carefully.

\section{Results}

Because of the experimenter-paced reading phase, subjects were asked whether they had enough time to read the first sentence of each pair. All subjects responded that they did, in fact, have sufficient time.

Of principal concern were the mean response latencies associated with the three experimental conditions. Subjects took $1,207,1,323$, and $1,288 \mathrm{msec}$ to respond to direct, inference, and control pairs, respectively. These differences were significant both with subjects $\left[F_{1}(2,66)=7.31, p<.025\right]$ and sentences $\left[F_{2}(2,34)=4.56, p<.025\right]$ treated as the random variable. Because the subjects random analysis revealed a significant list effect, the sentence random analysis was applied to response latencies from which the list effect had been removed.

Orthogonal comparisons were performed to examine questions central to this investigation. Response latencies for direct tests were faster than for the inference and control conditions combined $\left[F_{1}(1,66)=13.1\right.$, $\left.p<.01 ; \quad F_{2}(1,34)=13.0, p<.01\right]$. Inference and control latencies, however, did not differ significantly $\left[F_{1}(1,66)=1.26 ; F_{2}(1,34)=.88\right]$.

\section{Discussion}

Although the test items viewed in all conditions were identical, subjects took longer to indicate that they understood an item if it described an object to which no explicit mention was made in the antecedent. This difference cannot be attributed to the physical identity of the subject of the direct tests with a portion of their antecedents, as evidenced by the control condition latencies. The direct-inference latency difference of $116 \mathrm{msec}$ falls within the range of Haviland and Clark's (1974) measurements of 181,137 , and $74 \mathrm{msec}$ in several experiments.

These data are viewed as indicating that at least a subset of the cognitive processes associated with the inferences under examination are executed only when the test item is encountered. Implied instruments would seem, accordingly, not to have a status equivalent to that of explicit relations immediately after encoding.

It might be suggested that the subjects did compute and store the more highly probable inferences, but not the less probable ones. Averaging over all materials, one would still obtain the reported direct vs. inference latency difference. This suggestion can be answered by emphasizing that the antecedent sentences did not show much variation in the degree to which they suggested their corresponding instruments, as revealed by the norms described earlier.

There are other questions that arise in inspecting these results. First, it may be argued that the $2.5-\mathrm{sec}$ 
antecedent reading period is insufficient to permit the sort of inference activity being examined here. Second, there is the possibility that, as the testing session proceeds, subjects become increasingly aware that it would be useful to draw certain inferences. Such a tendency would not, of course, reflect ordinary language processes. If subjects behaved in this way, the latency difference between the inference and direct conditions would diminish with practice. The difference computed for the entire session would then underestimate inference time. Experiment 2 was designed to examine these questions.

\section{EXPERIMENT 2}

To explore the possibility that subjects in Experiment 1 had insufficient time to draw inferences from the antecedent sentences, Experiment 2 employed reading times of $2.5,4.0$, and $6.0 \mathrm{sec}$. The incompatibility of the 2.5 -sec period with inference computation would be reflected by the measurement of a Reading Time by Test Status (i.e., direct vs. inference) interaction: The direct-inference difference would diminish as reading time increased. Furthermore, within each level of test status, items were considered on the basis of whether they occurred in the first (Phase 1) or last (Phase 2) half of the list. If subjects draw more inferences in Phase 2 as a strategic ploy, inference time should be smaller in Phase 2 than in Phase 1. A Phase by Test Status interaction would then be obtained.

\section{Method}

From the 18 frames of Experiment 1, 16 were chosen at random. Two test lists were constructed. Half of the sentence sets appeared as direct items in List 1 and inference items in List 2, and vice versa for the other sets. Each list also included 16 filler pairs. The lists were constructed in such a way that exactly four direct, four inference, and eight filler items occurred in each half of the list. Another restriction prevented the appearance of more than two consecutive direct items or more than two consecutive inference items. The experimental lists were preceded by 14 practice pairs.

The subjects were 36 male and female undergraduate students from the same pool that was used for Experiment 1 . The subjects were randomly assigned to 1 of the 12 conditions described below, such that $\mathrm{n}=3$ for each condition.

The experiment employed a mixed design. The between subjects variables were reading time $(2.5,4.0$, or $6.0 \mathrm{sec})$, lis (1 or 2), and list order (forward or backward). The last variable was introduced so that each test item would occur in Phase 1 for some subjects and Phase 2 for others. There were, accordingly, 12 subject groups corresponding to the 3 by 2 by 2 crossing of reading time, list, and order. The within-subjects variables were test status (direct or inference) and phase (1 or 2 ).

During the test session, subjects responded to the 14 practice pairs followed by 32 experimental pairs. Practice was again not explicitly distinguished for the subjects. The procedure was identical to that of Experiment 1 except in that the antecedent sentence remained visible for $2.5,4.0$, or $6.0 \mathrm{sec}$.

\section{Results and Discussion}

The subjects once again confirmed that they always had enough time to read the antecedent sentences.
Analysis of variance was applied to the data, alternately employing subjects and sentences as the random variable. The analysis designating sentences as random was performed after the group effects were removed from the scores, as was the case in Experiment 1.

The only effects that reached significance for $\alpha=.05$ in both analyses were test status $\left[F_{1}(1,24)=7.25\right.$; $\left.F_{2}(1,15)=5.29\right]$, and phase $\left[F_{1}(1,24)=13.8 ; F_{2}(1,15)=\right.$ 5.30]. Inference latencies were $64 \mathrm{msec}$ longer than direct ones, and Phase 2 latencies were $68 \mathrm{msec}$ faster than those for Phase 1. Several interactions that were of particular interest, namely, Reading Time by Test Status, Phase by Test Status, and (perhaps) Reading Time by Phase by Test Status, did not approach significance (all Fs $<1$ in both analyses). Two four-way interactions that involved the order variable were significant in the subjects random analysis. No interpretation was made for the latter effects.

Brief mention should be made of the reading time variable. The means associated with this effect were $1,308,1,182$, and $1,315 \mathrm{msec}$, for the 2.5-, 4.0-, and 6.0-sec delays, respectively. While the subjects in the 4.0-sec condition responded more quickly, the effect did not approach significance $(F<1)$. More importantly, the test status effect was similar for all levels of delay. The response latencies associated with the 3 by 2 crossing of reading time and test status are shown in Figure 1.

As was the case in Experiment 1, response latencies for the inference condition exceeded the direct latencies. Neither of the other two hypotheses under examination received support. The invariance of the latency difference as a function of reading time indicates that the latency difference of Experiment 1 cannot be attributed to a lack of time for inference computation. The absence of a Phase by Test Status interaction suggests that subjects do not alter their encoding strategies as a function of practice.

To summarize briefly, the results of the first two experiments revealed that subjects needed more time to report understanding test sentences describing implicit instruments than those describing explicit ones. These results support the conclusion that subjects did not infer the use of the instruments in question while reading the antecedent sentences. The measured latency

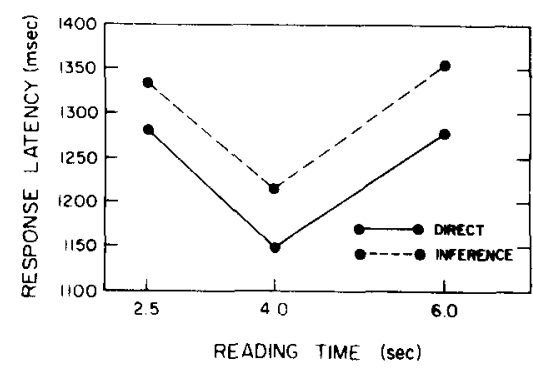

Figure 1. Mean response latencies (in milliseconds) of Experiment 2 for the direct and inference conditions as a function of reading time. 
difference between direct and inference tests is viewed as reflecting the additional mental processes required to reconcile (or "bridge"; see Clark, 1975) implicit instruments with the antecedent sentence. The additional activity corresponds to the computation of the instrument used to accomplish the act described in the antecedent.

Numerous investigators have, by now, argued that the reconciliation of each successive sentence in discourse with antecedent information is a necessary condition for comprehension (e.g., Carpenter \& Just, 1977; Thorndyke, 1976). The results of Experiments 1 and 2 indicate that Haviland and Clark's (1974) procedure may constitute a useful research tool for assessing the extent of processing that must occur to ensure such reconciliation. Underlying this proposal is the view that it is an oversimplification to view a proposition in discourse as simply supporting a particular inference or not. Rather, it is more reasonable to conceive of an inference continuum, which makes reference to the degree to which one proposition suggests or supports a related one.

In Experiment 3, subjects were asked to respond as soon as they understood test sentences that, according to the intuition of the experimenter, represented four degrees or levels of association with their antecedents. These levels included the direct and inference conditions of the first two experiments, plus two others that were viewed as even weaker than the former inference link. It was predicted that response latency would increase as the degree of association diminished.

\section{EXPERIMENT 3}

\footnotetext{
Method

The 16 sentence sets of Experiment 2 were expanded to include antecedents that were viewed as representing four degrees of association with their following test sentences. Sentences 6a-6e show the elaboration of Sentence Set 5 , discussed earlier.

Levels 1-4 are meant to represent, from greatest to least, the extent to which the antecedent suggests the use of a shovel. The four levels of antecedent, while certainly not defining an interval scale, were viewed as providing (1) a direct link, (2) a strong inferential link, (3) a weaker inferential link, and (4) at best, a very remote link. To feel that Sentence $6 \mathrm{e}$ had been understood in the context of $6 \mathrm{~d}$, for example, the subject would have to engage in some fairly elaborate mental computations, along the lines of the activities described by Thorndyke (1976). The subject might, for example, interpret Sentence 6d as suggesting that the bottles were broken, making the use of a shovel more plausible. Alternately, the demands of the task might cause the subject to register a response beyond some point in time, regardless of whether (s)he felt that the test item had been understood. In either case, the response latencies for Level 4 should be relatively high.

Four test lists were constructed, each of which consisted of 15 practice sentence pairs, followed by 16 experimental and 16 filler pairs. The latter 32 pairs were randomly interspersed. For each list, 4 of the 16 sets were assigned to each of the four conditions, in such a way that each set appeared in a different condition in each list.
}

The subjects were $28 \mathrm{male}$ and female introductory psychology summer students at the University of Manitoba. The subjects were randomly assigned to receive one of the four lists, so $\mathrm{n}=\mathbf{8}$ for each list. The procedure was identical to that of Experiment 1, with a $3.0-\mathrm{sec}$ experimenter-paced reading period.

\section{Results and Discussion}

The response latencies associated with the four levels of relation were $1,102,1,201,1,222$, and $1,342 \mathrm{msec}$ for Levels 1.4, respectively. Analysis of variance applied to these data, using the same plan as that described for Experiments 1 and 2, showed the "level" effect to be significant $\left[F_{1}(3,72)=10.1, p<.01 ; F_{2}(3,45)=12.0\right.$, $\mathrm{p}<.01]$. To examine the outcome more carefully, contrasts were applied to scores at every pair of adjacent levels. Only the contrast comparing the latencies for Level 2 vs. Level 3 relations failed to reach significance for $\alpha=.05\left[F_{1}(1,72)=.23 ; F_{2}(1,45)=.29\right]$. It should be noted that the difference between the two levels was in the expected direction: Subjects took $21 \mathrm{msec}$ longer to respond to Level 3 than to Level 2 test items.

The results, accordingly, support the notion that the present procedures reflect the extent of processing that is needed in order to achieve a "bridge" between a current sentence and an antecedent one. One possible limitation in this regard is related to the relatively small latency difference of $240 \mathrm{msec}$ between Level 1 and Level 4 pairs. What this implies is that the Haviland and Clark (1974) procedure may not clearly distinguist very many degrees of relationship. Some of the Level 4 antecedents did bear a vague relationship tc their following tests, as was discussed for Sentences $6 \mathrm{~d}$ and 6e. For others, such as "the clerk counted the money for the man" and "the string got tangled,' the relationship was remote indeed. Subjects chose nevertheless, to respond to Level 4 tests only $.24 \mathrm{sec}$ slower than for Level 1.

The absence of a significant latency difference between Level 2 and Level 3 latencies illustrates this limitation. While Level 3 antecedents were viewed as more weakly supporting the corresponding instrumenta inference than Level 2 antecedents, subjects needec only $21 \mathrm{msec}$ more for Level 3 tests.

The results reported by Just and Carpenter (in press, are very relevant to this point. In a study of inference activities in reading, they examined subjects' compre hension of sentence pairs that were very similar ir nature to the Levels 2 and 3 pairs from Experiment 3 The pair $7 \mathrm{a}-7 \mathrm{~b}$ illustrates a Level 2 relation (Just \& Carpenter, in press), while $8 \mathrm{a}-8 \mathrm{~b}$ illustrates Level 3 These pairs were embedded in five-sentence passage: for examination in two experiments. In the first, Jus and Carpenter asked their subjects to determine whethe: each successive sentence in the paragraph contradicter any of the previous information. In the second experi ment, a method similar to that of Haviland and Clark (1974) was used: Subjects were simply instructed ts 
respond when they understood each successive sentence. In the first experiment, the subjects, as predicted, needed (highly) significantly more time to respond to the second sentence of Level 3 than of Level 2 pairs (these sentences were never contradictory). In the second experiment, a difference in the expected direction was measured, but, as in the present Experiment 3, it did not reach significance. These results indicate that (1) Levels 2 and 3 pairs differ in nature, but (2) the Haviland and Clark procedure may not be adequate to bear this out.

\section{EXPERIMENT 4}

The fourth experiment was designed to address an alternative interpretation of the results of Experiment 1, and to extend the research to roles other than the instrumental. In Experiment 1, mean response latencies of $1,207,1,323$, and $1,288 \mathrm{msec}$ were measured for the direct, inference, and control conditions, respectively. Suppose that the direct antecedents provided an advantage of an appropriate "bridge" from test to antecedent, plus an advantage of encoding facilitation for the repeated lexical item. If it were the case that instrumental inferences are drawn during encoding, then the inference antecedents would provide only one of these advantages: namely, the appropriate bridge. Inference response latencies, accordingly, would still be longer than for the direct condition. Control latencies would also exceed direct ones, because these items would provide the encoding facilitation, and not the bridge.

To consider this interpretation, it was necessary to use a verification task rather than the Haviland and Clark (1974) procedure. Subjects read antecedent sentences of the sort employed in Experiments 1-3 and were asked to judge a test sentence true or false immediately after. The materials are illustrated by Sentence Set 9.

The antecedent-test sequences $9 \mathrm{a}-9 \mathrm{c}, 9 \mathrm{a}-9 \mathrm{~d}, 9 \mathrm{~b}-9 \mathrm{c}$, and $9 \mathrm{~b}-9 \mathrm{~d}$ represent the direct-true, direct-false, inferencetrue, and inference-false conditions, respectively. The important comparison is between the direct-false and inference-false conditions. For neither of these conditions does the antecedent sentence facilitate the encoding of the test. For this reason, if instrumental inferences were drawn during encoding, then response time would be the same for these two conditions. If not, then inference latencies should be longer than direct ones for the false as well as for the true condition.

The predictions of inference-during-encoding and inference-during-test models are shown in Table 2. Both models specify an additional encoding operation for all but the direct-true condition, and an additional falsification operation (Clark \& Chase, 1972) for both false conditions. The one discrepancy between the two approaches concerns possible additional inference activities for the inference-true and inference-false conditions and leads to the prediction of very different patterns of latencies. Only the inference-during-test model clearly indicates longer response latencies for inference-false than for direct-false. Both models predict a Test Status by Truth interaction, but the form of these interactions is quite different.

Suppose, furthermore, that the latency component associated with the extra encoding operation is very small. For the inference-during-test model, this would lead to the prediction of main effects of test status and truth but no interaction (see Table 2). For the inferenceduring-encoding model, only a main effect of truth would be predicted.

\section{Method}

Subjects. The subjects were 43 male and female undergraduates from the same pool as that employed for the other experiments. Sessions were conducted with groups of one to four individuals. Of the 43,1 person reported difficulty seeing the material, 2 did not understand the instructions, and the data of 2 were lost due to equipment failure. This left a sample of 38 subjects.

Materials. The verbal materials consisted of 36 sets of sentences like Set 9 . Exactly 12 of the sets were based on implications about each of the agent, patient, and instrument cases (Braine \& Wells, 1978; Fillmore, 1968). "The tooth was drilled," for example, implies the agent "dentist," and "the pitcher threw" suggests the patient "ball." Agent and patient sets are illustrated by Sentence Sets 10 and 11 , respectively. For both sets, the sequences $a-c, a-d, b-c$, and $b-d$ correspond to the direct-true, direct-false, inference-true, and inference-false conditions, respectively. The concepts filling the roles implied by the inference antecedents were first-choice elements agreed upon by an average of $82.9 \%$ of individuals. This figure is based on Singer's (Note 3) instrumental norms and additional norms collected for this study for the agent and patient cases.

The choice of the falsifying words merits some comment as well. For the agent and instrument sets, a list of secondchoice normed responses was prepared. "Principal," for example, was the second-choice agent for "scold the pupil." These items were then assigned randomly, within cases, as the falsifying

Table 2

Breakdown of Latencies Predicted by Inference-During-Encoding and Inference-During-Test Models

\begin{tabular}{|c|c|c|c|c|c|c|c|c|}
\hline \multirow[b]{2}{*}{ Condition } & \multicolumn{4}{|c|}{ Inference During Encoding } & \multicolumn{4}{|c|}{ Inference During Test } \\
\hline & Base & Encoding & Inference & Falsification & Base & Encoding & Inference & Falsification \\
\hline Direct-True & $\mathrm{b}$ & & & & $\mathrm{b}$ & & & \\
\hline Inference-True & $\mathrm{b}$ & $+e$ & & & $\mathrm{~b}$ & $+e$ & $+\mathrm{i}$ & \\
\hline Direct-False & b & te & & $+f$ & b & $+\mathrm{e}$ & & $+f$ \\
\hline Inference-False & $\mathrm{b}$ & $+e$ & & $+f$ & $\mathrm{~b}$ & $+\mathrm{e}$ & $+\mathbf{i}$ & $+f$ \\
\hline
\end{tabular}


items (e.g., "fill the tooth-principal"). For the patient case, this procedure often yielded sentences that included selectional restriction violations (Chomsky, 1965), such as "the tourists rowed the lottery." It was felt that subjects could judge these sentences to be false without carefully considering their meaning. For this reason, the falsifying words for the patient sets were the second-choice patients for these very phrases, based on the present norms. "Glove," for example, was the second-choice patient for "the pitcher threw."

The test sentences all used the active voice. In these sentences, the critical element (i.e., the implication or its explicit counterpart) was modified by the indefinite article "a" or "some." This was intended to designate the critical element as new, as opposed to given, information, and, accordingly, to induce the subjects to base their responses primarily upon the veridicality of the critical element (Clark \& Clark, 1977, Chapter 3).

Four test lists were constructed, each of which consisted of 36 antecedent-test sequences, preceded by four practice sequences. There were 12 sets associated with each of the agent, patient, and instrument cases. Within each list, 3 of the 12 sets for each case were assigned to each of the four experimental conditions in such a way that each set appeared in a different experimental condition in each list. The sets appeared in the same random order in the four lists. The order was created subject to several restrictions: First, of the nine examples for each condition (e.g., direct-true), either four were in the first half of the list and five in the second or vice versa. Second, exactly half of the 12 examples of each case occurred in each half of the list.

Procedure. The sessions were conducted with groups of one to four subjects. The subjects sat in separate rooms. The materials were displayed on 12-in. television screens at a distance of approximately $40 \mathrm{~cm}$ from the subject. The display of the sentences was controlled by a DEC PDP-8/a laboratory computer.

On each trial, a fixation point appeared on the screen for $2.5 \mathrm{sec}$. After $1 \mathrm{sec}$, the antecedent sentence appeared, beginning at the exact location of the fixation point and occupying two lines in the visual display. The antecedent remained visible for $2.5 \mathrm{sec}$ and was immediately replaced by the corresponding test sentence. The test sentence occupied only one line of display and remained visible for only $4 \mathrm{sec}$. The subjects were instructed to judge the truth of each test item in relation to its antecedent and to signal their responses by means of appropriately labeled keys in a response panel. They were asked to respond as quickly as possible without giving the wrong answer, and it was emphasized that they would be counted wrong if they did not respond during the 4-sec test presentation. Subjects' responses and response times were recorded automatically.

\section{Results}

It was discovered that the direct-true sequence of one of the patient sets was inadvertently omitted from its list and was replaced by another sequence from the same set. It was, therefore, necessary to estimate the direct-true latency for this set by averaging the directtrue scores of the other 11 patient sets. Likewise, in 6 out of 228 cases, a subject responded incorrectly to all three examples of a given experimental condition. Similar averaging remedies were employed to estimate these missing data.

The independent variables of interest were, particularly, test status, truth, and case. The mean latencies for correct trials were $1,926,2,167,2,302$, and $2,480 \mathrm{msec}$ for the direct-true, inference-true, direct- false, and inference-false conditions, respectively. Analysis of variance revealed main effects of test status $\left[F_{1}(1,30)=20.5 ; F_{2}(1,33)=18.9\right]$, truth $\left[F_{1}(1,30)=\right.$ $\left.38.1 ; \quad F_{2}(1,33)=69.7\right]$, and case $\left[F_{1}(2,60)=11.2\right.$; $\left.F_{2}(2,33)=4.76\right]$. All ps were less than .001 , except $\mathrm{p}\left(\mathrm{F}_{2}\right.$,case $)<.025$. The only significant interaction among these three variables was the Truth by Case interaction $\left[F_{1}(2,60)=12.6 ; F_{2}(2,33)=4.75 ;\right.$ ps $\left.<.025\right]$, the effect of truth being smaller for the instrumental case than for the others. The subjects random analysis also revealed several significant interactions involving the subject group variable, including Test Status by Group, Truth by Case by Group, and Test Status by Case by Group. The mean latencies associated with the four experimental conditions for each case are shown in Table 3.

Although the Test Status by Truth interaction was not significant, contrasts were performed to compare the direct-false and inference-false latencies. This was done because of the importance of this comparison. It was shown that latencies were significantly longer for the inference-false condition $\left[F_{1}(1,30)=19.7\right.$; $\left.\mathrm{F}_{2}(1,33)=6.74 ; \mathrm{ps}<.025\right]$

Errors and nonresponses. On each trial, the test sentence was displayed for only $4 \mathrm{sec}$, and subjects had to register a response during that time. Because subjects failed to respond on only $2.4 \%$ of the trials, nonresponses were combined with errors to obtain a measure of error rate. The overall error rate for the experiment was $11.0 \%$, reflecting rates of about $4 \%$, $15 \%, 8 \%$, and $17 \%$ for the direct-true, inference-true, direct-false, and inference-false conditions, respectively.

Two points need to be made about these values. First, the correlation between mean latency and error rate for the four conditions was $r=.74$, which discounts the possibility of a serious speed-accuracy tradeoff. Second, the high error rates for the inference conditions might be interpreted as reflecting a relatively high degree of disagreement on the part of subjects with regard to the appropriate implication for a given antecedent. Closer inspection of the data, however, seems to attribute the high error rates to confusion on the part of a small number of participants. Half of all the inferencefalse errors, for example, were committed by only 5 of 38 subjects. These five individuals, furthermore, had a mean correct inference-false latency of $2,545 \mathrm{msec}$, only $75 \mathrm{msec}$ higher than the mean of $2,470 \mathrm{msec}$ for

Table 3

Response Latencies for Experiment 4 as a Function of Test Status, Truth, and Case

\begin{tabular}{lccccc}
\hline & \multicolumn{2}{c}{ True } & & \multicolumn{2}{c}{ False } \\
\cline { 5 - 6 } \cline { 5 - 5 } Case & Direct & Inference & & Direct & Inference \\
\hline Agent & 1948 & 2225 & 2433 & 2648 \\
Patient & 1979 & 2147 & 2349 & 2606 \\
Instrument & 1850 & 2130 & 2126 & 2187 \\
\hline
\end{tabular}


the other 33 subjects. While these subjects may have misunderstood the instructions, it does not seem likely that the overall latency pattern was seriously distorted by their scores.

\section{Discussion}

The results are viewed as strongly supporting the position that the inferences in question were not drawn during the encoding of the antecedents. If they had been, then direct- and inference-false latencies should have been approximately equal. Inference-false latencies, however, were significantly longer than those of the direct-false condition.

The latency pattern, in fact, matches quite closely the predictions of that version of the inference-duringtest model that assumes that the encoding facilitation effect is very small. The fit of this model is especially good for the agent and patient. It is only for the false instrument materials that the size of "inference time" is not well over $100 \mathrm{msec}$.

The psychological reality and usefulness of different cases has been inspected by several investigators (e.g., Braine \& Wells, 1978; Dosher, 1976; Shafto, 1973). Shafto (1973), for example, suggested that judgments about the roles underlying a sentence (or proposition) are based upon the dimensions "active-passive" and "living-nonliving." In this framework, for example, the instrument case is active and nonliving. Such analyses have raised the possibility that the language recipient might be more likely to draw inferences about some cases than others. The absence of a Test Status by Truth by Case interaction for Experiment 4 , however, suggests similar inference processing for the three cases examined.

The analysis also revealed a main effect of case. The latencies for the instrument tests $(2,073 \mathrm{msec})$ were somewhat smaller than for the other two cases $(2,292 \mathrm{msec})$, but this was probably due to the fact that all instrument tests were of the form "the [agent] used a [instrument]," repeating the verb "used" each time. For the agent and patient, the test items had the form "the/a [agent] [verb] a/the [patient]." Despite this difference in form, similar latency patterns were obtained for all three cases.

These results answer yet another interpretation of Experiment 1. Recall that the actions of Experiment 1 had first-choice instrument ratings of about $85 \%$. Suppose that subjects did draw instrumental inferences while reading the antecedent. It is conceivable, in this case, that $15 \%$ of them could have computed an inference other than the one embedded in the test sentence. The longer response latencies for inference than for direct tests might then have been based upon the $85 \%$ of trials for which the correct inference was drawn and response latency was, hypothetically, the same as for direct trials, and the $15 \%$ of trials for which the wrong inference was drawn and the subjects had to correct their inferences at test time, a process that might be very time consuming. If this position were correct, however, direct-false and inference-false response latencies should have been the same. This contradicts the results of Experiment 4.

\section{GENERAL DISCUSSION}

Four experiments were conducted to explore the question of whether the language recipient computes certain inferences during the course of sentence encoding. It was argued that if the inferences in question are so computed, subjects should need no more time to understand test sentences that express an implication of an antecedent than for others that refer to a portion of the antecedent directly. In Experiment 1, however, subjects needed more time for inference than for direct tests. Experiment 2 revealed that this effect can be measured for antecedent reading times of $2.5,4.0$, and $6.0 \mathrm{sec}$, and that it does not change with practice within the test session. Experiment 3 demonstrated that this procedure can be employed to distinguish more than two degrees of relationship between antecedent and test. Experiment 4 extended the findings to the agent and patient cases. The results indicate that the internal representation of sentences does not include, with the status of explicit ideas, implicit ideas of the sort studied here.

The case-filling inferences examined here fall within the framework of what Clark (1977) called "indirect reference by characterization: inducible roles." This term refers generally to probabilistic (or pragmatic; see Harris \& Monaco, 1978) case-filling inferences. The question may be raised, therefore, whether similar findings might be obtained in the investigation of other inference classes described by Clark (1977). Do language recipients, for example, compute the existence of windows ("inducible parts") when a room is being discussed? It may be speculated that they do not, but further evidence is clearly needed. Equally interesting is the question of whether "necessary" (as contrasted with "inducible"; see Clark, 1977) roles are automatically computed. While "clearing snow," for example, only strongly suggests the use of a shovel, "the millionaire was murdered" logically implies the existence of a killer.

In general, further research concerning these problems must identify the linguistic and psychological factors that guide inference activity in the course of comprehension. One suggestion concerning these factors can be derived from the procedure employed in Experiments 1-3. These experiments were designed to probe the issue of whether certain inferences are drawn in the course of comprehension. The subjects were presented with test sentences that required that the inferences under investigation be computed if they had not been drawn during the encoding of the antecedent. 
If sentences like the present test sentences can induce inference computation in a laboratory setting, it is conceivable that this could be true in more natural language environments. Clark (1975) has referred to inferences that function so as to achieve a link between the present phrase and the earlier discourse as backward inferences. In contrast, "forward inference" would refer to the situation in which, in the terminology of the present study, an inference is drawn during the encoding of the antecedent. It may be possible to show that much of the inference activity of natural language comprehension occurs in situations demanding a backward inference. Studies by McKoon and Keenan (1974) and Thorndyke (1976) already provide some evidence that backward inferences do occur.

\section{REFERENCE NOTES}

1. Paris, S. G., Sorkin, J. R., \& Pisoni, D. B. The role of implied instruments in sentence memory. Paper presented at the Annual Meeting of the Midwestern Psychological Association, Chicago, May 1974.

2. Singer, M. The temporal locus of inference in language comprehension. University of Manitoba, 1976.

3. Singer, M. Inferences about instruments: Response norms. University of Manitoba, 1977. (Available from M. Singer, Department of Psychology, University of Manitoba, Winnipeg R3T 2N2. Canada.)

\section{REFERENCES}

Anderson, R. C., \& Ortony, A. On putting apples into bottles-A problem of polysemy. Cognitive Psychology, 1975 , 7. 167-180.

Barclay. J. R., Bransford, J. D., Franks, J. J., McCarrell, N. S., \& Nirsch. K. Comprehension and semantic flexibility. Journal of Verbal Learning and Verbal Behavior, 1974, 13, 471-481.

Braine, M. D. S., \& Wells, R. S. Case-like categories in children: The actor and some related categories. Cognitive Psychology, 1978, 10, 100-122.

Carpenter, P. A., \& JusT, M. A. Integrative processes in comprehension. In D. Laberge \& S. J. Samuels (Eds.), Perception and comprehension. Hillsdale. N.J: Erlbaum, 1977.

Cномsкy. N. Aspects of the theory of syntax. Cambridge, Mass: MIT. 1965.

Clark, H. H. Bridging. In R. Schank \& B. Nash-Webber (Eds.), Theoretical issues in natural language processing. Cambridge, Mass: MIT. Interdisciplinary Workshop, 1975.

CLARK, H. H. Inferences in comprehension. In D. Laberge \& S. J. Samuels (Eds.), Perception and comprehension. Hillsdale, N.J: Erlbaum, 1977.

Clark. H. H., \& Chase, W. G. On the process of comparing sentences against pictures. Cognitive Psychology, 1972, 3, $472-517$.

Clark. H. H.. \& Clark, E. V. Psychology and language. New York: Harcourt, Brace, \& Jovanovich, 1977.

Corbett. A. T., \& Dosher, B. A. Instrument inferences in sentence encoding. Journal of Verbal Learning and Verbal Behavior. in press.
Dosher, B. A. The retrieval of sentences from memory: A speed-accuracy study. Cognitive Psychology, 1976, 8, 291-310.

Fill.more, C. The case for case. In E. Bach \& R. Harms (Eds.), Universals in linguistic theory. New York: Holt, Rinehart, \& Winston, 1968.

HARRIS, R. J. Memory and comprehension of implications and inferences of complex sentences. Journal of Verbal Learning and Verbal Behavior, 1974, 13, 626-637.

Harris, R. J., \& Monaco, G. E. The psychology of pragmatic implication: Information processing between the lines. Journal of Experimental Psychology: General, 1978, 5, 1-22.

Haviland, S. E., \& Clark, H. H. What's new? Acquiring new information as a process in comprehension. Journal of Verbal Learning and Verbal Behavior, 1974, 13, 512-521.

Hayes-Roth, B., \& Hayes-Roth, F. The prominence of lexical information in memory representations of meaning. Journal of Verbal Learning and Verbal Behavior, 1977, 16, 119-136.

Johnson, M. K., Bransford, J. D., \& Solomon, S. K. Memory for tacit implications of sentences. Journal of Experimental Psychology, 1973, 98, 203-205.

Just, M. A., \& CARPENTER, P. A. The relation between comprehending and remembering some complex sentences. Memory \& Cognition, 1976, 4, 318-322.

Just, M. A., \& CARPenter, P. A. Inference processes during reading: Reflections from eye fixations. In J. W. Senders $\&$ R. A. Monty (Eds.), Eye movements and the higher psychological functions. Hillsdale, N.J: Erlbaum, in press.

McKoon, G., \& Keenan, J. M. Response latencies to explicit statements as a function of the delay between reading and test. In W. Kintsch (Ed.). The representation of meaning in memory. Hillsdale, N.J: Erlbaum, 1974.

Paris, S., \& Lindauer. B. K. The role of inference in children's comprehension and memory for sentences. Cognitive Psychology, $1976,8,217-227$.

Sнағто, M. The space for case. Journal of Verbal Learning and Verbal Behavior, 1973, 12, 551-562.

Thorndyke, P. W. The role of inferences in discourse comprehension. Journal of Verbal Learning and Verbal Behavior, 1976, $15,437.446$.

TILl, R. E. Sentence memory prompted with inferential recall cues. Journal of Experimental Psychology: Human Learning and Memory, 1977, 3, 129-141.

Tulving, E., \& Thompson, D. M. Encoding specificity and retrieval processes in episodic memory. Psychological Review, $1973,80,352-373$.

\section{NOTE}

1. It might be objected that the norms of Singer (Note 3) do not provide enough information about the materials of Experiment 1 . The norms show, for example, that $95.5 \%$ of subjects agreed upon "hammer" as the first-choice response for "drive the nail." It is clear that equivalent norms could be collected for whole sentences. The agreement about "hammer" might be less for "the native drove the nail" than for "the worker drove the nail." In Experiment 1, every effort was made to name agents and locations that would not lead to unusual interpretations of the corresponding actions.

(Accepted for publication February 9, 1979.) 